\title{
A aprendizagem em temas de geociências mediada pela aplicação de mapas conceituais
}

Os mapas conceituais (MC) têm se destacado na atualidade como uma ferramenta poderosa no processo da aprendizagem em diversas áreas do conhecimento e níveis e ensino. O objetivo deste trabalho foi aplicar os MC como recurso didático no processo de ensino-aprendizagem em conteúdo de geociências. O estudo foi realizado com 33 alunos do 70 ano do ensino fundamental II de uma escola privada de natal, RN. As atividades foram desenvolvidas por meio das seguintes etapas: I) aprofundamento temático (estrutura dos vulcões e erupções vulcânicas), II) familiarização com os MC, e, III) elaboração dos MC. Os mapas construídos foram avaliados quanto ao tipo morfológico (radial, linear e em rede), utilizando a classificação de Kinchin et al. (2000). Partindo dos resultados observados nesse estudo, verificou-se uma grande ocorrência de mapas com estrutura em rede $(58 \%)$ o que evidencia que os estudantes aprenderam de forma significativa os temas abordados. Por meio dos dados obtidos, verificamos que o uso de MC contribui para o desenvolvimento de habilidades e da capacidade dos estudantes em organizar, relacionar e representar o conhecimento que foi construído, podendo assim, ser utilizado como recurso didático no processo de ensino e aprendizagem em temas de geociências.

Palavras-chave: Organizador Gráfico; Mapas Conceituais; Ensino de Geociências; Educação Básica.

\section{Learning on geoscience themes mediated by the application of concept maps}

Concept maps (CM) have stood out today as a powerful tool in the learning process in several areas of knowledge and levels and teaching. The objective of this work was to apply CM as a didactic resource in the teaching-learning process in geoscience content. The study was carried out with 33 students from the 7th year of elementary school II at a private school in Natal, RN. The activities were developed through the following steps: I) thematic deepening (structure of the volcanoes and volcanic eruptions), II) familiarization with the MC, and, III) elaboration of the MC. The constructed maps were evaluated for morphological type (radial, linear and network), using the classification of Kinchin et al. (2000). Based on the results observed in this study, there was a great occurrence of maps with a network structure (58\%), which shows that students have significantly learned the topics covered. Through the data obtained, we found that the use of CM contributes to the development of skills and the ability of students to organize, relate and represent the knowledge that was built, thus being able to be used as a didactic resource in the teaching and learning process in geoscience themes.

Keywords: Graphic Organizer; Concept Maps; Teaching of Geosciences; Basic Education

\section{Topic: Ciência do Solo}

Reviewed anonymously in the process of blind peer
Received: $\mathbf{1 0 / 0 7 / 2 0 2 0}$

Approved: $22 / 10 / 2020$
Clécio Danilo Dias da Silva

Universidade Federal do Rio Grande do Norte, Brasil

http://lattes.cnpq.br/4235157508528733

http://orcid.org/0000-0002-7776-8830

danilodiass18@ufrn.edu.br
Referencing this:

SILVA, C. D. D.. A aprendizagem em temas de geociências mediada pela aplicação de mapas conceituais. Natural Resources, v.10, n.3, p.1-7, 2020. DOI: http://doi.org/10.6008/CBPC22379290.2020.003.0001 


\section{INTRODUÇÃO}

O ensino de Geociências tem como objetivo entender as dinâmicas do sistema terra por meio da investigação das relações existentes entre suas diferentes esferas (atmosfera, biosfera, litosfera, hidrosfera e noosfera); desde os fenômenos visíveis até os que não podemos ver, mas que podemos interferir (SILVA, 2014; FIRMINO et al., 2019). Trata-se de um ensino interdisciplinar que ultrapassa as atividades de conhecer, identificar, classificar, reconhecer agentes, atores, fatos, locais e acontecimentos que descrevam a terra em sua totalidade estrutural, física, química, biológica e humana (GARCIA et al., 2014). Logo, ensinar Geociências envolve o desenvolvimento de raciocínios, processos de estabelecimento de relações, avaliação e análise de fenômenos que estão em constante interação no planeta, desde sua formação até hoje (CARNEIRO et al., 2012; MARTINS et al., 2014).

Além de explorar e correlacionar tópicos comuns as áreas de Ciências e Geografia, o ensino das Geociências torna-se relevante por levantar questões extremamente atuais, ligadas a sustentabilidade do planeta versus recursos naturais disponíveis, pois o homem, além de ocupar e "sobreviver" no ambiente, também o explora e degrada de maneira exacerbada (CARNEIRO et al., 2004; PIRANHA et al., 2009). Nesse contexto, a sua inserção na educação básica pode contribuir para formar cidadãos críticos, que atuem sobre o sistema Terra e compreendam as consequências de sua atuação, desenvolvendo consciência ambiental para a conservação da natureza (GARCIA et al., 2014). Entretanto, Toledo (2005) afirma que no desenvolvimento integral no ensino das Geociências verifica-se que os alunos possuem conhecimentos privados que não são considerados suficientes para adquirir uma visão de funcionamento global e interdependente da natureza. Dessa forma, correm o risco de desenvolverem uma visão imediatista e utilitária da natureza, enquanto meio físico que proporciona soluções às necessidades modernas humanas de materiais e energia, e que também proporciona problemas de degradação, que não são compreendidos como respostas naturais às ações de interferência nos ciclos naturais (COMPIANI, 2010; CARNEIRO et al., 2012).

No que diz respeito aos Mapas Conceituais (MC), Moreira (2010) os conceituam como diagramas indicando relações entre conceitos, ou entre palavras que usamos para representar conceitos. Para Kinchin (2010), os MC são ferramentas gráficas que possibilitam a organização do conhecimento, e visam representar as relações significativas na forma de preposição. Conforme Kinchin (2014) eles são reconhecidos mundialmente por contribuir na qualidade da aprendizagem dos alunos, e foram recebidos positivamente nos diversos níveis de escolaridade. Em relação às formas em que os $\mathrm{MC}$ podem ser usados na prática docente, Moreira (2010) aponta que eles são uteis para mostrar relações significativas entre conceitos ensinados em uma única aula, em uma unidade de estudo ou em um curso inteiro. De modo geral, eles se tornam elementos de grande importância a serem desenvolvidos ao longo de uma sequência didática mobilizadora de aprendizagem, e de construção do conhecimento (SILVA et al., 2019).

Considerando os MC como uma ferramenta de aprendizagem, Novak et al. (2010) destacam como utilidades destes para os alunos nas seguintes formas: resolver problemas; síntese dos conteúdos vistos em 
sala; planejar o estudo; preparar-se para avaliações; perceber as relações entre as ideias de um dado conteúdo; fazer anotações, entre outros. Para Correia et al. (2016), os MC ainda podem possibilitar outros aspectos positivos em sala de aula, como: organização do conhecimento e estudo, revisão, avaliação, socialização e colaboração.

No tocante ao ensino de Geociências, os MC vêm mostrando resultados positivos no processo de ensino-aprendizagem em diversas temáticas, como por exemplo: camadas da atmosfera, propriedades do ar, biomas brasileiros, aguas continentais, estrutura da terra, bioacumulação de poluentes, paleontologia, entre outros (MOURA, 2014, TOMITA et al., 2016; SILVA et al., 2018; FIRMINO et al., 2019). Diante deste contexto, este trabalho teve como objetivo aplicar os mapas conceituais como recurso didático no processo da aprendizagem em temas de geociências na educação básica, evidenciando a estrutura básica dos vulcões e tipos de erupções vulcânicas.

\section{METODOLOGIA}

O presente estudo é caracterizado como pesquisa qualitativa, com procedimentos direcionados à análise de conteúdo. A pesquisa qualitativa é um estudo de determinado fato, objeto, situação, grupo de pessoas ou fenômenos da realidade. Ela tem sido amplamente utilizada em pesquisas educacionais pois possibilita ao pesquisador a compreender a realidade em que está inserido em sua total dimensão e complexidade. No que se refere a análise de conteúdo, ela possibilita ao pesquisador examinar os dados da pesquisa através de inferências que utilizam indicadores e se configura desde a elaboração dos cálculos que fornecem dados, até a extração de estruturas traduzíveis.

\section{Aplicação das atividades desenvolvidas}

O estudo foi realizado com 33 alunos do 70 ano do ensino fundamental II de uma escola privada de Natal, RN. As atividades foram desenvolvidas em 03 etapas (Quadro 1).

Quadro 1: Detalhamentos das etapas do estudo.

\begin{tabular}{|c|l|l|l|l|}
\hline \multicolumn{2}{|l|}{ Etapas } & Objetivos & Materiais utilizados & Duração \\
\hline 1 & $\begin{array}{l}\text { Aprofundamento } \\
\text { temático }\end{array}$ & $\begin{array}{l}\text { Aprofundar os conhecimentos dos estudantes quanto a } \\
\text { estrutura dos vulcões, funcionamento e tipos de } \\
\text { erupções. }\end{array}$ & $\begin{array}{l}\text { Datashow, slides e } \\
\text { documentário. }\end{array}$ & $\begin{array}{l}3 \\
\text { horas/aulas } \\
(100 \text { min) }\end{array}$ \\
\hline 2 & $\begin{array}{l}\text { Familiarização com } \\
\text { os MC }\end{array}$ & $\begin{array}{l}\text { Possibilitar aos estudantes um contato prévio com a } \\
\text { ferramenta de ensino a ser utilizada nas aulas. }\end{array}$ & $\begin{array}{l}\text { Roteiro de normas sobre } \\
\text { os MC em folha A4. }\end{array}$ & $\begin{array}{l}1 \text { hora/aula } \\
(100 \text { min) }\end{array}$ \\
\hline 3 & Elaboração de MC & $\begin{array}{l}\text { Avaliar os conhecimentos dos estudantes sobre a } \\
\text { temática proposta por meio dos conceitos externalizados } \\
\text { e tipologia estrutural dos MC. }\end{array}$ & Folhas A4. & $\begin{array}{l}2 \\
\text { horas/aulas } \\
(100 \text { min) }\end{array}$ \\
\hline
\end{tabular}

Inicialmente, houve um aprofundamento temático por meio de aulas expositivas e dialogadas sobre os conteúdos 'estrutura dos vulcões' e as 'erupções vulcânicas'. Para complementar este momento, foi aplicado e discutido em sala de aula o documentário 'Vulcões: poder e devastação' da Discovery Channel (44 min). Em seguida, foi realizada uma familiarização com os $M C$ por meio de leituras e discussões contendo normas, passos, e dicas de como elaborar bons mapas conceituais, conforme instruções de Aguiar et al. (2013). Posteriormente, houve a elaboração de MC, onde os estudantes receberam uma folha A4 e foram 
instruídos a elaborarem $01 \mathrm{MC}$ para o conteúdo trabalhado dentro do aprofundamento temático.

\section{Avaliação dos materiais elaborados}

Para avaliar os MC, buscou-se a frequência dos principais conceitos presentes nos materiais elaborados. Para identificar indícios da aprendizagem dos estudantes, utilizou-se o tipo morfológico do MC construído, conforme a classificação de Kinchin et al. (2000).

Os mapas foram categorizados nos seguintes tipos morfológicos (Quadro 2): I) radial: onde se expõe pouco domínio sobre o tema. Este tipo de mapa apresenta proposições a partir do conceito central/raiz, e estes, não estão diretamente ligados uns aos outros; II) linear, no qual se visualiza algum domínio sobre o tema. Mapas com essa estrutura apresenta um encadeamento sequencial dos conceitos. Embora possam apresentar uma natureza hierárquica, muitas vezes, as proposições podem ser inapropriadas, isto é, conceitualmente erradas; III) em rede, onde se verifica alto domínio do tema. Apresenta uma boa estrutura hierárquica, com uma rede conceitual altamente integrada e inter-relacionada.

Quadro 2: Tipos morfológicos de MC e correspondências com entendimento de conteúdo.

\begin{tabular}{|l|l|l|}
\hline $\begin{array}{l}\text { Mapa Radial } \\
\begin{array}{l}\text { Possivelmente tem pouco domínio sobre } \\
\text { otema }\end{array}\end{array}$ & $\begin{array}{l}\text { Mapa Linear } \\
\text { Possivelmente tem algum domínio sobre o } \\
\text { tema }\end{array}$ & $\begin{array}{l}\text { Mapa em Rede } \\
\text { Possivelmente tem um alto domínio do } \\
\text { tema. }\end{array}$ \\
\hline
\end{tabular}

Fonte: Adaptado de Kinchin et al. (2014).

Os dados foram sistematizados em planilhas e processados no software Microsoft Excel 2010, para a análise dos resultados e representação em quadros e tabelas.

\section{RESULTADOS E DISCUSSÃO}

Conforme Moreira (2010) a análise dos mapas é essencialmente qualitativa. Para o autor deve-se "procurar interpretar a informação dada pelo aluno no mapa a fim de obter evidências de aprendizagem significativa" (MOREIRA, 2010), aliados a estes elementos, encontra-se a classificação em tipos morfológicos, propostos por Kinchin et al. (2000), a qual é capaz de identificar traços de aprendizagens conforme o tipo de MC construído pelo estudante.

Dentro deste contexto, avaliou-se a estrutura morfológica dos $\mathrm{MC}$, onde se constatou que dos $33 \mathrm{MC}$ elaborados pelos alunos, 6 apresentaram estrutura radial (18\%), 8 possuíam estrutura linear (24\%), enquanto a estrutura em rede correspondeu a 19 mapas (58\%). Exemplos de mapas conceituais ${ }^{1}$ construídos pelos estudantes com estrutura radial (E24), linear (E11) e em rede (E9) podem ser visualizadas na Figura 1.

\footnotetext{
${ }^{1}$ Os MCs da Figura 1, foram elaborados pelos estudantes dentro da sequência de atividades em folha A4. Entretanto, foram passados para o software Cmap tools ${ }^{\circledast}$ (IHMC, 2018). A forma e os elementos utilizados (linhas, setas, palavras de ligação) utilizados pelos estudantes foram respeitados, ou seja, não foram feitas alterações/modificações.
} 


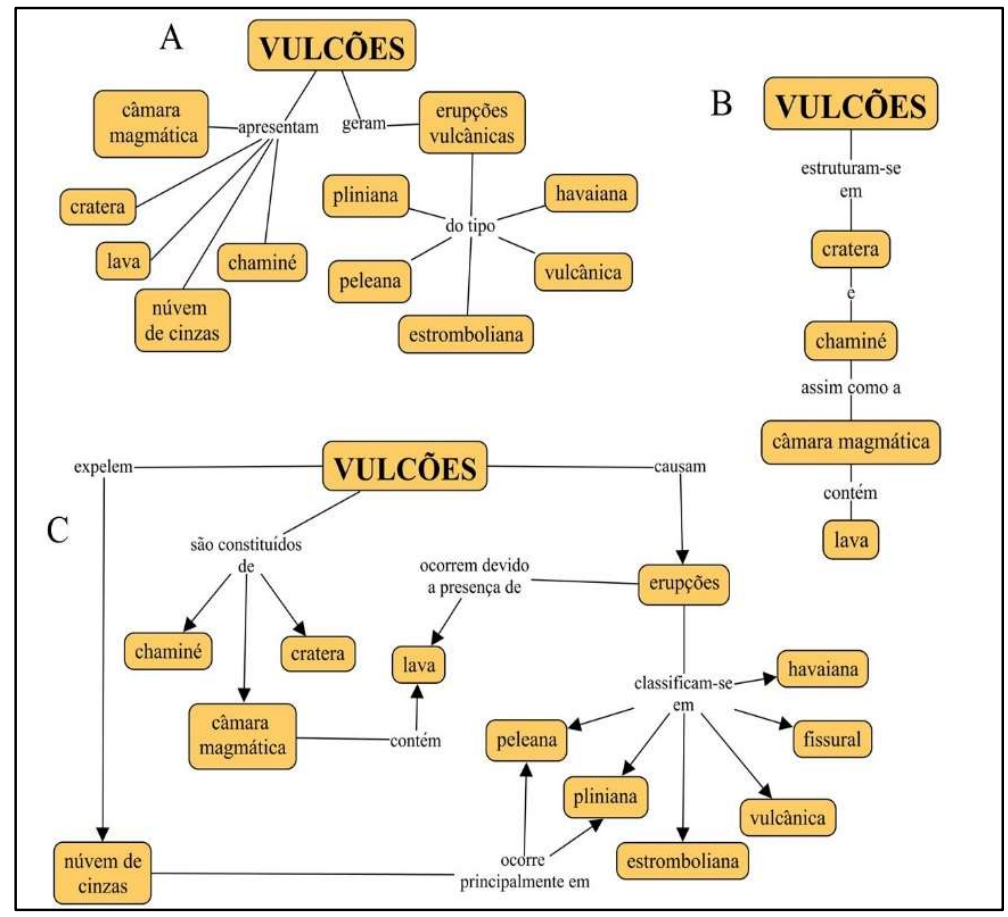

Figura 2: Exemplos de MC elaborados pelos estudantes com as estruturas A) radial, B) linear, C) em rede.

Os conceitos e suas respectivas frequências estão detalhados no Quadro 3. Sobre a estrutura básica dos vulcões, os conceitos mais frequentes nos mapas foram 'câmara magmática', 'chaminé', 'cratera' presentes nos $33 \mathrm{MC}$ avaliados, o equivalente a 100\%. O conceito 'Lava' estava contido em 28 MC, representando $85 \%$. Com menor frequência encontrou-se o conceito 'nuvem de cinzas' presentes 18 MC, o equivalente a $55 \%$.

Quadro 3: Conceitos sobre os vulcões presentes nos MC elaborados pelos estudantes.

\begin{tabular}{|l|l|l|l|l|l|}
\hline Estrutura dos vulcões & Freq. & $\%$ & Tipos de erupções & Creq. \\
\hline Conceitos & 33 & $100 \%$ & E. Havaiana & 26 & $79 \%$ \\
\hline Câmara magmática & 33 & $100 \%$ & E. Vulcânica & 26 & $79 \%$ \\
\hline Chaminé & 33 & $100 \%$ & E. Estrombliana & 22 & $67 \%$ \\
\hline Cratera & 28 & $85 \%$ & E. Fissural & 22 & $67 \%$ \\
\hline Lava & 18 & $55 \%$ & E. Peleana & 20 & $61 \%$ \\
\hline Nuvem de cinzas & - & - & E. Pliniana & 20 & $61 \%$ \\
\hline- & & & & \\
\hline
\end{tabular}

Com relação aos tipos de erupções, diagnosticou-se uma grande frequência para os termos 'erupção havaiana' e 'erupção vulcânica', ambos, presentes em 26 MC, correspondentes a 79\%. Logo, seguiram-se os conceitos 'erupção fissural' e 'erupção estrombiliana', presentes em $22 \mathrm{MC}$ representadas em 67\%, e, com menor frequência estavam presentes os termos 'erupções peleana' e 'erupções lliniana' em 20 MC, equivalendo a $61 \%$ (Tabela 1 ).

Conforme Correia et al. (2016) a estrutura de um MC, pode caracterizar a estrutura cognitiva do aprendiz e trazer evidências de uma aprendizagem significativa. Dessa forma, levando em consideração a grande frequência de mapas com estrutura em rede (58\%), podemos considerar que houve indícios de uma aprendizagem profunda e significativa por meio dos MC para os assuntos abordados. Resultados semelhantes foram encontrados por Santos (2019), que ao analisarem MC de estudantes do ensino fundamental em temas de geociências, identificaram uma grande frequência de mapas com estrutura em rede, assim como na 
presente pesquisa. Em síntese, Aguiar et al. (2013) afirmam que "somente alunos que aprendem um tema de forma significativa são capazes de produzir um MC em "rede", enquanto MC "radiais" e "lineares" são persistentes durante o processo educativo dos alunos que optaram pela aprendizagem mecânica".

\section{CONCLUSÕES}

Diante dos dados observados nesse estudo, verificou-se uma grande ocorrência de mapas conceituais com estrutura em rede, o que evidencia que os estudantes aprenderam o tema abordado de forma significativa, sendo constatados por meio da grande quantidade de conceitos inseridos nos MC envolvendo a estrutura do vulcão e tipos de erupções. De modo geral, os MC são ferramentas focadas nos estudantes e não no professor. Eles contribuem com o desenvolvimento de habilidades e da capacidade dos alunos em organizar e representar o conhecimento que possui através da disposição e interligação de conceitos. Dentro deste contexto, os MC podem ser utilizados como instrumento de ensino e aprendizagem em temas de geociências na educação básica.

\section{REFERÊNCIAS}

AGUIAR, J. G.; CORREIA, P. R. M.. Como fazer bons mapas conceituais? Estabelecendo parâmetros de referências e propondo atividades de treinamento. Revista Brasileira de Pesquisa em Educação em Ciências, v.13, n.2, p.141-157, 2013.

CARNEIRO, C. D. R.; TOLEDO, M. C. M.; ALMEIDA, F. F. M.. Dez motivos para a inclusão de temas de Geologia na Educação Básica. Revista Brasileira de Geociências, v.34, n.4, p.553-560, 2004

CARNEIRO, C. D. R.; SANTOS, G. R. B.. Ensino de geociências na formação profissional em meio ambiente no estado de São Paulo. Revista Brasileira de Geociências, v.42, n.1, p.8495, 2012. DOI: http://dx.doi.org/10.5327/Z037575362012000500008

COMPIANI, M.. Narrativas e desenhos no ensino de astronomia/geociências com o tema "a formação do universo": um olhar das geociências. Revista Ensaio, v.12, n.02 p.257-278, 2010.

CORREIA, P. R. M.; AGUIAR, J. G.; VIANA, A. D.; CABRAL, G. C. $P$.. Por que vale a pena usar mapas conceituais no ensino superior?. Revista de Graduação USP, v.1, n.1, p.41-51, 2016. DOI: https://doi.org/10.11606/issn.2525376X.v1i1p41-51

FIRMINO, A. R. S, BARBOSA, J. R. A.; RODRIGUES, A. P. C.. Ensino de geociências no ensino fundamental: um estudo de caso sobre uso de mapas conceituais e aulas práticas (RJBrasil). Experiências em Ensino de Ciências, v.14, n.2, p.272291, 2019.

GARCIA, C. B.; IMBERNOM, R. A. L.; LACERDA, L. A. V.. Desenvolvimento de recursos didáticos para o ensino de Geociências para a Banca das Ciências e Experimentoteca da EACH/USP. Terræ Didática, v.10, n.3, p.331-335, 2014.
KINCHIN, I. M.. Concept mapping as a learning tool in higher education: a critical analysis of recent reviews. The Journal of Continuing Higher Education, v.62, n.1, p.39-49, 2014.

KINCHIN, I. M.; STREATFIELD, D.; HAY, D. B.. Using concept mapping to enhance the research interview. International Journal of Qualitative Methods, v.9, n.1, p.52-68, 2010.

MARTINS, J. R. S.; CARNEIRO, C. D. R.. Método científico e controvérsias nas Geociências. Terræ Didática, v.10, n.3, p.240-249, 2014

DOI: https://doi.org/10.20396/td.v10i3.8637330.

MOREIRA, M. A.. Mapas conceituais como instrumentos para promover a diferenciação conceitual progressiva e a reconciliação integrativa. Ciência e Cultura, v.32, n.4, p.474479, 2010.

MOURA, R. W. S.. As caatingas do Cariri Paraibano: mapa conceitual como ferramenta para aprendizagem significativa no ensino de biologia. Dissertação (Mestrado em Educação) - Universidade Federal da Paraíba, João Pessoa, 2014.

NOVAK, J. D.; CAÑAS, A. J.. A teoria subjacente aos mapas conceituais e como elaborá-los e usá-los. Práxis Educativa, v.5, n.1, p.9-29, 2010 DOI: https://doi.org/10.5212/PraxEduc.v.5i1.009029

PIRANHA, J. M.; CARNEIRO, C. D. R.. O ensino de geologia como instrumento formador de uma cultura de sustentabilidade. Revista Brasileira de Geociências, v.39, p.129-137, 2009.

SANTOS, D. B.. Os mapas conceituais como ferramenta avaliativa no processo da aprendizagem em temas de geociências. In: CONGRESSO NACIONAL DE PESQUISA E ENSINO EM CIÊNCIAS, 4. Anais. Campina grande Editora Realize, 2019. 
SILVA, D. F.. Formação de professores em associação à institucionalização do ensino de Geociências no Brasil (19732014). In: SEMINÁRIO NACIONAL DE HISTÓRIA DA CIÊNCIA E DA TECNOLOGIA, 14. Anais. Belo Horizonte: SNHCT, 2014.

SILVA, R. V. B.; ALMEIDA, C. M.; PORTO, M. D.. Teaching of geociences: paleontology and geology an approach based on significant learning. Revista Mirante, v.11, n.7, p.106-120, 2018.

SILVA, C. D. D.; SANTOS, R. L.; D'OLIVEIRA, R. G.; ALMEIDA, E. A.. Motivações de estudantes para aprendizagem em zoologia por meio de mapas conceituais. Brazilian Journal of Development, v.5, n.11, p.26715-26734, 2019. DOI:

https://doi.org/10.34117/bjdv5n11-298

TOLEDO, M. C. M.. Geociências no Ensino Médio Brasileiro Análise dos Parâmetros Curriculares Nacionais. Revista do Instituto de Geociências, v.3, p.31-44, 2005.

TOMITA, L. M. S.; TORRES, E. C.; FONSECA, R. L.. O uso de mapas conceituais como facilitadores da aprendizagem significativa: a bacia hidrográfica em foco. Boletim Paulista de Geografia, n.94, p.47-64, 2016.

A CBPC - Companhia Brasileira de Produção Científica (CNPJ: 11.221.422/0001-03) detém os direitos materiais desta publicação. Os direitos referem-se à publicação do trabalho em qualquer parte do mundo, incluindo os direitos às renovações, expansões e disseminações da contribuição, bem como outros direitos subsidiários. Todos os trabalhos publicados eletronicamente poderão posteriormente ser publicados em coletâneas impressas sob coordenação da Sustenere Publishing, da Companhia Brasileira de Produção Científica e seus parceiros autorizados. Os (as) autores (as) preservam os direitos autorais, mas não têm permissão para a publicação da contribuição em outro meio, impresso ou digital, em português ou em tradução. 